\title{
Serum lactate dehydrogenase as a biochemical marker for maternal outcome in pre-eclampsia
}

\author{
Gitanjali Kumari*, Vaishali Taralekar, Suchita Dabhadakr
}

Department of Obstetrics and Gynecology, Bharati Vidyapeeth (Deemed to be University) Medical College, Pune, Maharashtra, India

Received: 30 September 2021

Revised: 30 October 2021

Accepted: 01 November 2021

*Correspondence:

Dr. Gitanjali Kumari,

E-mail: gitanjali.kumari9@gmail.com

Copyright: (c) the author(s), publisher and licensee Medip Academy. This is an open-access article distributed under the terms of the Creative Commons Attribution Non-Commercial License, which permits unrestricted non-commercial

\begin{abstract}
Background: LDH is a cytoplasmic intracellular enzyme present in the heart, kidney, muscle, leukocytes and erythrocytes, of all major organ systems. The presence of LDH in extracellular space points towards cellular damage, endothelial dysfunction. Preeclampsia is a multisystem disorder during pregnancy causing cellular damage or death. Hence, serum LDH levels can be helpful in determining the extent of cell damage and the seriousness of this disease. The present study aimed to correlate the maternal serum lactate dehydrogenase levels with maternal and perinatal outcomes in women with pre-eclampsia.

Methods: It was a prospective observational study. A total of 120 antenatal patients diagnosed with hypertensive disorder of pregnancy were included in this study. Serum LDH levels were estimated by enzymatic method on the autoanalyzer. Patients were grouped into 3 categories according to serum LDH levels: a) <600 IU/l, b) 600-800 IU/l c) $>800$ IU/1. Clinical manifestation of development of complications of hypertensive disease and its relation with serum LDH in respective patients were analyzed.

Results: An LDH level of more than 800 IU/l was seen in $19.2 \%$ while between 600 to 800 IU/l was seen in $16.7 \%$ cases. A significant association was observed between incidence of maternal complications with high LDH levels $(\mathrm{p}<0.01)$. High LDH levels were observed to be associated with development of ante-partum haemorrhage, eclampsia and requirement of ICU admission. No significant association was found between different categories of LDH and deep tendon reflexes, levels of proteinuria at the time of admission in these preeclamptic women.

Conclusions: Close monitoring and early intervention of the preeclampsia patients with elevated serum LDH levels can help avoid adverse effects of the disease and thereby help improve maternal and perinatal outcomes in pregnant women with preeclampsia.
\end{abstract}

Keywords: Hypertensive disorders of pregnancy, Intra uterine growth retardation, Lactate dehydrogenase, Pre eclampsia

\section{INTRODUCTION}

Preeclampsia is a multiorgan system disorder with multifactorial aetiology. It is defined by blood pressure of more than $140 / 90 \mathrm{mmHg}$ on two separate occasions separated by 4-6 hours apart associated with proteinuria. It affects around $5-8 \%$ of all pregnancies. ${ }^{1}$ The incidence is affected by parity, age and race, ethnicity, etc. A hospitalbased study was conducted in India in 2006, in which the incidence of hypertensive disorder of pregnancy was $5.38 \%$, out of which preeclampsia, eclampsia and HELLP syndrome were $44 \%, 40 \%$ and $7 \%$ respectively and of chronic hypertension and chronic hypertension with superimposed preeclampsia were $9 \%$ in total. ${ }^{2}$ Preeclampsia is associated with maternal and perinatal morbidity and mortality and other various complications such as DIC, HELLP, abruption, eclampsia etc. ${ }^{2-4}$ 
LDH levels have recently been proposed as possible indicators for predicting the severity of pre-eclampsia and as a multi-organ involvement predictor. ${ }^{5}$

LDH is a cytoplasmic intracellular enzyme present in the heart, kidney, muscle, leukocytes and erythrocytes, of all major organ systems. The presence of LDH in extracellular space points towards cellular damage, endothelial dysfunction. Preeclampsia is a multisystem disorder causing cellular damage or death. Hence, serum LDH levels can be helpful in determining the extent of cell damage and the seriousness of the disease. The complications of Preeclampsia such as abruption of placenta, failure of kidney and liver dysfunction and cardiovascular collapse could be life threatening. Thus, combining various biomarkers especially those related to vascular damage like lactate dehydrogenase may be helpful in determining the risk of occurrence of preeclampsia and thus may help in preventing it. ${ }^{5,6}$

So, this research aimed to compare the levels of maternal serum lactate dehydrogenase with the maternal and perinatal outcomes of pre-eclampsia women.

\section{METHODS}

This observational and clinical prospective study was conducted in a tertiary care medical college hospital (Bharati hospital) in Maharashtra during October 2018October 2020.

\section{Inclusion criteria}

It included 120 consecutive pregnant women with a singleton pregnancy diagnosed with pre-eclampsia (hypertensive disorder of pregnancy).

\section{Exclusion criteria}

Antenatal women with chronic hypertension; pre-existing diabetes mellitus, renal disease, liver disorder, thyroid disorder were excluded from the study.

After obtaining informed and written consent, the detailed history and thorough clinical examination of all participants were done. All women were subjected to routine antenatal investigations. Serum LDH levels were estimated by enzymatic method on the autoanalyzer. Patients were grouped into 3 categories according to serum LDH levels: a) less than $600 \mathrm{IU} / \mathrm{l}$, b) between $600-800$ IU/l, c) more than $800 \mathrm{IU} / 1$.

These patients were followed up for the development of premonitory signs and symptoms like epigastric pain, blurred visions, vomiting, and headache. Depending upon the premonitory signs and symptoms, blood pressure, and investigations, they were categorized as mild and severe pre-eclampsia and patients were managed as per hospital protocol.
Observations were recorded about maternal outcomes such as eclampsia/HELLP syndrome/abruptio placenta/ICU admission/intracranial haemorrhage/acute renal failure/ pulmonary edema/DIC.

Gestational age wise comparative analysis was done for LDH levels with maternal outcomes. Gestation age groups were as follows: a) 20-28 weeks of gestation, b) 29-34 weeks of gestation, c) $>34$ weeks of gestation.

\section{RESULTS}

It was observed that patients were uniformly distributed with respect to mean age of patients, parity and mean gestational age among various LDH categories in preeclampsia cases. In our study mean age of patients with preeclampsia was 27.98 years with $63.3 \%$ cases between 20-30 years of age. In present the study, a total of $39.2 \%$ cases of pre-eclampsia were prim gravida while $60.8 \%$ were multigravida.

Table 1: Distribution of study cases as per lactate dehydrogenase (LDH) levels.

\begin{tabular}{|lll|}
\hline LDH levels (IU/I) & N & Percentage \\
\hline$<600$ & 77 & 64.2 \\
\hline $\mathbf{6 0 0 - 8 0 0}$ & 20 & 16.7 \\
\hline$>\mathbf{8 0 0}$ & 23 & 19.2 \\
\hline Total & 120 & 100.0 \\
\hline
\end{tabular}

It was observed that serum LDH level of more than 800 IU/l was seen in $19.2 \%$ while between 600 to $800 \mathrm{IU} / 1$ was seen in $16.7 \%$ cases (Table 1 ).

Overall maternal complication rate among cases of preeclampsia was $23.4 \%$ in the present study. The Most common complication associated with cases of preeclampsia was ante-partum haemorrhage (13.3\%) followed by development of eclampsia (2.5\%). HELLP syndrome developed in 1 case $(0.8 \%)$. No complication was reported in $76.6 \%$ of cases.

Incidence of caesarean section was significantly higher in cases with high LDH levels $(\mathrm{p}<0.01)$. Incidence of emergency C-section was $72.7 \%, 30 \%$ and $49.4 \%$ in LDH levels of $>800 \mathrm{IU} / 1,600-800 \mathrm{IU} / 1$ and $<600 \mathrm{IU} / 1$ respectively. A significant association was noted between incidence of maternal complications with high LDH levels $(\mathrm{p}<0.01)$. Incidence of maternal complications was $52.5 \%$, $15 \%$ and $19.5 \%$ in LDH levels of $>800 \mathrm{IU} / 1,600-800 \mathrm{IU} / 1$ and $<600 \mathrm{IU} / 1$ respectively. High LDH levels were observed to be associated with development of antepartum haemorrhage, eclampsia and requirement of ICU admission (Table 2). No association was observed between incidence of thrombocytopenia, proteinuria, deep tendon reflex and LDH levels in pre-eclampsia cases (p-0.185). 
Table 2: Association of LDH levels with type of maternal complications.

\begin{tabular}{|c|c|c|c|c|c|}
\hline \multirow[b]{2}{*}{ Maternal complications } & \multicolumn{3}{|c|}{ LDH (IU/I) } & \multirow{2}{*}{ Total } & \multirow[b]{2}{*}{$P$ value } \\
\hline & $<600$ & $600-800$ & $>800$ & & \\
\hline \multirow{2}{*}{$\begin{array}{l}\text { Haemorrhage } \\
\text { (APH/PPH) }\end{array}$} & 9 & 1 & 6 & 16 & \multirow{2}{*}{0.09} \\
\hline & $11.7 \%$ & $5.0 \%$ & $26.1 \%$ & $13.3 \%$ & \\
\hline \multirow{2}{*}{ ICU admission } & 2 & 0 & 3 & 5 & \multirow{2}{*}{0.05} \\
\hline & $2.6 \%$ & $0.0 \%$ & $13.0 \%$ & $4.2 \%$ & \\
\hline \multirow{2}{*}{ Eclampsia } & 1 & 2 & 0 & 3 & \multirow{2}{*}{0.06} \\
\hline & $1.3 \%$ & $10.0 \%$ & $0.0 \%$ & $2.5 \%$ & \\
\hline \multirow{2}{*}{ Ascites } & 0 & 0 & 2 & 2 & \multirow{2}{*}{0.01} \\
\hline & $0.0 \%$ & $0.0 \%$ & $8.7 \%$ & $1.7 \%$ & \\
\hline \multirow{2}{*}{ HELLP } & 0 & 0 & 1 & 1 & \multirow{2}{*}{0.12} \\
\hline & $0.0 \%$ & $0.0 \%$ & $4.3 \%$ & $0.8 \%$ & \\
\hline \multirow{2}{*}{ LSCS } & 44 & 14 & 16 & 74 & \multirow{2}{*}{0.05} \\
\hline & $30 \%$ & $42.9 \%$ & $70 \%$ & $61.66 \%$ & \\
\hline
\end{tabular}

\section{DISCUSSION}

Preeclampsia is defined as a syndrome unique to pregnancy that can have a virtual effect on every organ system of the body. It is responsible for systemic endothelial dysfunction which in turn causes vasoconstriction and decreased oxygen saturation (hypoxia) thus resulting in MODS (multiple organ dysfunction syndromes). Multiple studies and researches have been conducted in the past to determine a biochemical marker that can serve as a determinant for prognosis and to assess the severity of preeclampsia for e.g. LDH, homocysteine, serum calcium, calcium/creatinine ratio etc. In this study, complications of preeclampsia in antenatal women and their perinatal outcome are studies with respect to serum $\mathrm{LDH}$ levels.

In our study we found that the patients were uniformly distributed with respect to mean age, mean gestational age and parity in various categories of preeclampsia. In research conducted by Nadkarni et al the age group of women with hypertensive disorder of pregnancy was between $21-25$ years. $^{7-9}$

\section{Mode of delivery}

Vaginal delivery was possible in only a third of the cases $(38.3 \%)$ in present study while caesarean section was done in $61.7 \%$ cases. In research conducted by Nadkarni et al, the number of LSCS was higher in preeclampsia group than compared to normotensive pregnant women. ${ }^{9}$ Kheir et al in their study observed that nearly all cases $(98.5 \%)$ were delivered by caesarean section. ${ }^{8}$

\section{Type of delivery}

In the present study, the mean gestation age for delivery was 33.7 weeks with only a third of the cases (34.2\%) delivered at term ( $\geq 37$ weeks) and $65.8 \%$ delivered preterm.
Nadkarni et al in their study observed that significantly more cases of eclampsia delivered pre-term $(61.6 \%)$ as compared to controls $(24.3 \%) .{ }^{9}$ Similar results were also observed by Kheir et al, where high incidence $(52 \%)$ of preterm delivery was seen in cases with pre-eclampsia. ${ }^{8}$

\section{Maternal complications}

Overall maternal complication rate among cases of preeclampsia was $23.4 \%$ noted in the present study correlates with findings from previous studies e.g. Payne et al, studied a total of 2081 cases of Hypertensive disorders of pregnancy (HDP). ${ }^{8,9}$ The adverse outcome was observed in $401(19.26 \%)$ of these females. Similarly, in a another study, 12.9 and $18.3 \%$ were percentage of adverse outcomes in women with hypertensive disorders of pregnancy respectively. ${ }^{10}$

\section{CONCLUSION}

Thus, to summarize, serum LDH is a reliable biochemical prognostic marker for predicting the prognosis in pregnant women with preeclampsia, for predicting the severity of the disease and the maternal complications associated with it. Hence serum LDH levels must be done in all pregnant women with preeclampsia at their first visit to a health centre.

Funding: No funding sources

Conflict of interest: None declared

Ethical approval: The study was approved by the Institutional Ethics Committee Bharati Vidyapeeth Deemed to be University Medical College

\section{REFERENCES}

1. Report of National High Blood Pressure Education Program Working Group on High Blood Pressure in Pregnancy. Am J Obstet Gynecol. 2000;183:S1-22. 
2. Prakash J, Pandey LK, Singh AK, Kar B. Hypertension in pregnancy: hospital based study. J Assoc Phys India. 2006;54:273-8.

3. Barton JR, O’Brien JM, Bergauer NK, Jacques DL, Sibai BM. Mild gestational hypertension remote from term: progression and outcome. Am J Obstet Gynecol. 2001;184(5):979-83.

4. Sibai BM, Sarinoglu C, Mercer BM. Eclampsia. VII. Pregnancy outcome after eclampsia and long-term prognosis. Am J Obstet Gynecol. 1992;166(6 Pt 1):1757-63.

5. Maynard SE, Min JY, Merchan J, Lim KH, Li J, Mondal S, et al. Excess placental soluble fms-like tyrosine kinase 1 (sFlt1) may contribute to endothelial dysfunction, hypertension, and proteinuria in preeclampsia. J Clin Invest. 2003;111(5):649-58.

6. Qublan H, Ammarin V, Bataineh O, Al-Shraideh Z, Tahat Y, Awamleh I, et al. LDH as biochemical marker of adverse pregnancy outcome in severe preeclampsia. Med Sci Monit. 2005;11:393-7.

7. Matthys LA, Coppage KH, Lambers DS, Barton JR, Sibai BM. Delayed postpartum preeclampsia: an experience of 151 cases. Am J Obstet Gynecol. 2004;190(5):1464-6.

8. Kheir A. Neonatal Outcome in hypertensive disorders of pregnancy in a tertiary neonatal unit in Sudan. $\mathbf{J}$ Med Med Res. 2014;2(5):59-65.

9. Nadkarni J, Bahl J, Parekh P. Perinatal outcome in pregnancy associated hypertension. Indian Pediatr 2001;38:174-8.

10. Payne BA, Hutcheon JA, Ansermino JM, Hall DR, Bhutta ZA, Bhutta SZ, et al. A risk prediction model for the assessment and triage of women with hypertensive disorders of pregnancy in low-resourced settings: the miniPIERS (Pre-eclampsia Integrated Estimate of RiSk) multi-country prospective cohort study. PLoS Med. 2014;11(1):e1001589.

Cite this article as: Kumari G, Taralekar V, Dabhadakr S. Serum lactate dehydrogenase as a biochemical marker for maternal outcome in preeclampsia. Int J Reprod Contracept Obstet Gynecol 2021;10:4541-4. 